\title{
LAS MEDIDAS CAUTELARES ANTICIPATORIAS E INNOMINADAS EN EL PROCESO ARBITRAL EN COLOMBIA*
}

\author{
ANTICIPATORY PRECAUTIONARY \\ MEASURES AND INNOMINATE IN THE \\ ARBITRATION PROCESS IN COLOMBIA
}

Cindy Charlotte Reyes-Sinisterra**

Fecha de recepción: 26 de julio de 2015 Fecha de aceptación: 21 de enero de 2016 Disponible en linea: 30 de mayo de 2016

\section{Para citar este artículo/To cite this article}

Reyes-Sinisterra, Cindy Charlotte, Las medidas cautelares anticipatorias e innominadas en el proceso arbitral en Colombia, 132 Vniversitas, 389-422 (2016). http://dx.doi.org/10.11144/Javeriana.vj132.mcai

doi:10.11144/Javeriana.vj132.mcai

* Este artículo de investigación es producto parcial del proyecto Implicaciones del Código General del Proceso en el Proceso Arbitral en Colombia, el cual hace parte del Grupo de Investigación en Derecho Procesal de la Universidad Libre de Cúcuta.

** Abogada de la Universidad Libre, Seccional Cúcuta. Especialista en derecho procesal civil, Universidad Externado de Colombia. MSc en derecho procesal contemporáneo, Universidad de Medellín. Docente de las materias teoría general del proceso, derecho procesal civil general, derecho comercial general, títulos valores y prueba electrónica, Universidad Libre de Cúcuta. Ganadora del IX Concurso Internacional de Estudiantes de Derecho, organizado por el Instituto Colombiano de Derecho Procesal. Abogada litigante en asuntos civiles y comerciales. Asesora y consultora empresarial. Directora de los proyectos La Prueba Electrónica en Colombia, La costumbre mercantil válida en Cúcuta, e Incidencias de la entrada en vigencia del Código General del Proceso en el proceso arbitral en Colombia (Ley 1563 de 2012), como docente investigadora y colíder del grupo de investigación de Derecho Procesal GIDPRO, Universidad Libre de Cúcuta. Actualmente, directora encargada del Centro Seccional de Investigaciones, Universidad Libre de Cúcuta. El estudiante Christian Camilo Gómez participó en este artículo como auxiliar de investigación. Contacto: cindy.reyes@ unilibrecucuta.edu.co 


\section{RESUMEN}

Con la adopción del Estatuto Nacional de Arbitraje Nacional e Internacional, Colombia dio un gran paso en materia de arbitraje. Los aportes de la Ley 1563 de 2012 son novedosos frente a lo que había antes en el país, en especial en materia de medidas cautelares de diferentes tipos, es decir, nominadas e innominadas. Sin embargo, es necesario hacer referencia a que la solicitud, el decreto y la práctica de estas medidas se pueden dar en dos momentos: antes y después de ser notificado el demandado en un proceso arbitral. Sin embargo, la Ley 1563 de 2012 presenta un vacío en cuanto a la adopción de las denominadas medidas cautelares anticipadas, por lo que, de acuerdo a lo que se puede conocer como las implicaciones del Código General del Proceso en el proceso arbitral en Colombia, esta investigación se desarrolla con base en el arbitraje internacional, para que en el país, se regule esta figura y así garantizar que en el proceso arbitral se tutelen de forma efectiva los derechos reconocidos por la ley sustancial, tal como se concibe en el Código General del Proceso y en la Constitución Política de Colombia. Esta investigación hace referencia a figuras como los árbitros de urgencia, el proceso prearbitral y la teoría del apoyo judicial a los árbitros como un catálogo abierto de posibilidades que se presentarán frente a expertos en el tema que adopten la mejor opción para que el arbitraje nacional se adecúe a la implementación de las medidas cautelares anticipatorias (nominadas e innominadas) en el proceso arbitral en Colombia.

Palabras clave: Medidas cautelares innominadas; medidas cautelares anticipadas; tutela judicial; árbitros de emergencia; proceso prearbitral; arbitraje nacional; arbitraje internacional

\section{ABSTRACT}

With the adoption of the national status of domestic and international arbitration, Colombia took a big step in arbitration, the contributions of the Law 1563 of 2012 are novel for arbitration, especially regarding precautionary measures of different types, i.e., nominated and nameless. However, it is necessary to refer to the application, recital and practice of these measures can be taken in two periods: before and after being notified by the defendant in an arbitration process. However, the 1563 Act of 2012 presents a gap in the adoption of precautionary measures called early, so the Hotbed of Litigation in this paper makes a proposal based on international arbitration, to be regulated in Colombia this figure and so ensure that the arbitration process will safeguard effectively the rights recognized by substantive law, as envisaged in the General Code of Procedure and the Constitution of Colombia. This research figures such as the arbiters of urgency, the pre-arbitration and judicial support the theory of the referees as an open catalog of possibilities will be presented in front of experts in the field to take the best option refers to domestic 
arbitration is appropriate to the implementation of precautionary measures and anticipatory unnamed in the arbitration process in Colombia.

Keywords: Precautionary Measures unnamed; Advance Precautionary Measures; Tutela Legal; Referees Emergency Pre-Arbitral Referee Procedure; National Arbitration; International Arbitration

\section{SUMARIO}

Introducción.- Metodología.- I. Las medidas cautelares.- A. Generalidades.- 1. Las medidas cautelares en el proceso arbitral: visión mundial.- 2. Las medidas cautelares en el proceso arbitral colombiano.- 3. Las medidas cautelares anticipatorias e innominadas.- a. Las medidas cautelares anticipatorias. Generalidades.- b. Concepto. Las medidas cautelares anticipatorias según Sartori.- II. ANTECEDENTES DE LAS MEDIDAS CAUTELARES ANTICIPATORIAS E INNOMINADAS EN EL PROCESO ARBITRAL INTERNACIONAL. - A. Antecedentes de las medidas cautelares anticipatorias en el arbitraje internacional.- 1 . Asociación de arbitraje francesa.- 2. Referee prearbitral de la CCI.- 3. Optional Rules for Emergency Measures of Protection, American Arbitration Association, AAA.- 4. Emergency Relief Rules, Organización Mundial de la Propiedad Intelectual, OMPI.- 5. Associazione Italiana per l'Arbitrato.- 6. Centro Empresarial de Conciliación y Arbitraje, CEDCA.- B. Antecedentes de las medidas cautelares innominadas en el proceso arbitral internacional.- 1 . Las medidas cautelares innominadas en España.- 2. Las medidas cautelares innominadas en Inglaterra y Suecia.- 3. Reglamento de Arbitraje de la CCI (Cámara de Comercio Internacional).- 4. Reglamento de Arbitraje de la American Arbitration Association, AAA.- 5. Reglamento del Tribunal Arbitral de la Organización Mundial de la Propiedad Intelectual, OMPI.- 6. Reglamento de arbitraje de la London Court of International Arbitration, LCIA.- III. LAS MEDIDAS CAUTELARES ANTICIPATORIAS EN EL proceso arbitral en Colombia.- A. Aspectos críticos.- 1. Solicitud.- 2. Decreto.- 3. Práctica.- B. Propuesta.- 1. Medidas cautelares anticipatorias en el proceso arbitral en Colombia.- a. Solicitud.- b. Decreto.- c. Práctica.Conclusiones.- Bibliografía. 


\section{INTRODUCCIÓN}

Las providencias cautelares representan una conciliación entre las dos exigencias, frecuentemente opuestas de la justicia: la de la celeridad y la ponderación; entre hacer las cosas pronto pero mal, y hacerlas bien pero tarde...

\section{Piero Calamandrei}

El arbitraje en lenguaje común evoca la facultad de arbitrar, presumiendo de garantizar el cumplimiento de unas reglas preestablecidas para la resolución de diferencias entre dos o más personas ${ }^{1}$. En pocas palabras, se puede decir: "El arbitraje es un instrumento a través del cual un tercero, denominado árbitro, revestido con determinados poderes, resuelve un conflicto de intereses, que recae sobre materias de libre disposición mediante un auto definitivo, denominado laudo, que adquiere vinculatoriedad e inmutabilidad en un ordenamiento jurídico"\%"

De otro lado, como mecanismos para garantizar la efectividad de un derecho que sea objeto de litigio, aparecen las medidas cautelares. En Colombia, a partir de la entrada en vigencia del Código General del Proceso el 1 de enero de 2016, pueden ser de varios tipos: nominadas e innominadas, ya sean anticipatorias o dentro del proceso.

Para desarrollar uno de los aspectos que se estudian en el proyecto de investigación, del cual este artículo es un producto parcial, se quiere hacer un análisis teórico-práctico acerca de las vicisitudes que se presentan respecto de la solicitud, decreto y práctica de las medidas cautelares innominadas y anticipatorias en el proceso arbitral en Colombia. Es decir, se hará un análisis integral de Ley 1563 de $2012^{3}$ o Estatuto Arbitral en Colombia, y la Ley 1564 de

1 Luis Fraga-Pittaluga, El arbitraje en el derecho administrativo, 29-30 (Los Talleres Gráficos de Editorial Torino, Caracas, 2000).

2 En su mayoría, las legislaciones arbitrales del mundo, sea cual fuese la tradición jurídica a la que pertenecen, reconocen que los laudos arbitrales adquieren la firmeza que permite su ejecutabilidad y prohíbe iniciar un nuevo proceso o arbitraje entre las mismas partes y sobre el mismo objeto conocido en el arbitraje ya concluido. Bernardo M. Cremades \& Ignacio Madalena, Procedimientos paralelos en el arbitraje internacional, 6 Revista Peruana de Arbitraje, 3-88, 38-39 (2008). Disponible en: http://www.peruarbitraje.org/pdf/revista/ REVISTA_PERUANA_DE_ARBITRAJE_RPA_6_2008.pdf

3 Colombia, Ley 1563 de 2012, por medio de la cual se expide el Estatuto de Arbitraje Nacional e Internacional y se dictan otras disposiciones, 48.489 Diario Oficial, 12 de julio de 2012. Disponible en: http://www.secretariasenado.gov.co/senado/basedoc/ley_1563_2012.html 
2012 o Código General del Proceso ${ }^{4}$, que se abordará primero a partir de los conceptos y generalidades que presentan las medidas cautelares anticipatorias e innominadas tanto en Colombia, como en el arbitraje internacional, y el estudio de los aspectos críticos frente a la solicitud, decreto y práctica de las medidas cautelares anticipatorias e innominadas en el proceso arbitral en el ámbito nacional, para plantear una propuesta a partir de lo investigado y finalmente, exponer las respectivas conclusiones.

Mientras a la comunidad académica puede parecerle claro el avance que representan las regulaciones mencionadas anteriormente, hay mucho por decir respecto de la facultad que como árbitros pueden tener los particulares para decretar medidas cautelares innominadas y además anticipatorias en lo que se pueda entender como un proceso arbitral, por cuanto se puede observar que el arbitraje es un mecanismo alternativo para resolver litigios sin necesidad de acudir a la jurisdicción ordinaria, es decir, por medio de este, un particular administra justicia de forma transitoria, pues las partes que lo habilitaron para esto se someterán de igual forma a su decisión, por lo que constituye un mecanismo heterocompositivo, pues "el arbitraje posee tal calidad, aun cuando el árbitro que juzga es designado por las partes, no implica que sean estas y no aquel el que decide la forma como debe resolverse el conflicto, el árbitro y el juez, es aquel tercero imparcial, llamado a resolver, y es el quien finalmente lo hace"5.

\section{Metodología}

El tipo de estudio de esta investigación es teórico-analítico. Es teórico, porque se estudiarán la legislación, los autores, la jurisprudencia, el derecho comparado, los reglamentos de arbitraje nacional e internacional y la doctrina en torno a las medidas cautelares anticipatorias e innominadas en el proceso arbitral en Colombia y en otros países que son referentes en materia arbitral, partiendo de temáticas generales, por cuanto hasta el momento no se ha iden-

4 Colombia, Ley 1564 de 2012, por medio de la cual se expide el Código General del Proceso y se dictan otras disposiciones, 48.489 Diario Oficial, 12 de julio de 2012. Disponible en: http:// www.secretariasenado.gov.co/senado/basedoc/ley_1564_2012.html

5 Fernando Reglero-Campos, El arbitraje: el convenio arbitral y las causas de nulidad del laudo en la ley de 5 de diciembre de 1988, 29 (Montecorvo, Madrid, 1991). 
tificado ningún estudio teórico que de forma específica mencione la aplicación de este tipo de medidas cautelares prearbitrales e innominadas en el proceso arbitral colombiano en vigencia de la Ley 1563 de 2012, o Estatuto Nacional e Internacional de Arbitraje.

\section{LAS MEDIDAS CAUTELARES}

\section{A. Generalidades}

Las medidas cautelares son herramientas que protegen de manera provisional la integridad de un derecho, para evitar la aparición de riesgos en los procedimientos jurídicos. Su principal objetivo es prevenir un peligro o un daño en los procesos jurisdiccionales y garantizar la eficacia de estos. Nacen en el derecho privado interno de los estados y posteriormente se trasladan al derecho internacional público, para proteger los derechos en los distintos procedimientos. En Colombia, las medidas cautelares conllevan una decisión jurisdiccional proferida ya sea por los jueces o por los particulares en los casos excepcionales previstos en la Constitución, para asegurar la efectividad de un derecho que posteriormente será objeto de litigio.

\section{Las medidas cautelares en el proceso arbitral: visión mundial}

Según Marcela Rodríguez-Mejía: "En la esfera internacional comparada hay países que no contemplan en sus normativas internas que los árbitros tengan competencia en materia cautelar, otorgando la exclusividad de la misma a los jueces. Pueden servir como ejemplo, sin ánimo de exhaustividad, el caso de Italia, China, Argentina, Honduras y Brasil"'.

6 Marcela Rodríguez-Mejía, Medidas cautelares en el proceso arbitral (Editorial Externado de Colombia, Bogotá, 2013). 


\section{Las medidas cautelares en el proceso arbitral colombiano}

Según Rafael Bernal Gutiérrez, en Colombia en aplicación de la Ley 1563 de 2012: "para adoptar medidas cautelares, tiene el Tribunal que seguir un marco de referencia que debe ponderar cuidadosamente. El solicitante deberá demostrar al Tribunal Arbitral, la conducencia, pertinencia, razonabilidad, y oportunidad de la medida cautelar"?

\section{Las medidas cautelares anticipatorias e innominadas}

\section{a. Las medidas cautelares anticipatorias. Generalidades}

"Las medidas innovativas o anticipatorias del resultado de la estimación de la pretensión, como mecanismo más idóneo para que las partes participen en el proceso en igualdad de condiciones, produciendo una innovación sobre la situación jurídica preexistente al proceso principal..." .

\section{b. Concepto. Las medidas cautelares anticipatorias según Sartori}

"Tratándose de cautelares innovativas, estas contienen un auténtico juicio de mérito. La resolución provisoria recae directamente sobre la relación sustancial controvertida, constituyendo una declaración interina sobre el fondo (por ejemplo, el derecho a los alimentos provisorios, art. 375 Código Civil [argentino]). Al respecto, la CSJN [Corte Suprema de Justicia de la Nación, Argentina] sostuvo que dentro de las medidas cautelares, la innovativa es una decisión excepcional porque altera el Estado de derecho existente al tiempo de su dictado, habida cuenta [de] que configura un anticipo de jurisdicción favorable respecto al fallo final de la causa, lo que justifica una mayor prudencia en la apreciación de los recaudos que hacen a su admisión; y por su intermedio se intenta aventar, más

7 Marcela Rodríguez-Mejía, Medidas cautelares en el proceso arbitral (Editorial Externado de Colombia, Bogotá, 2013).

8 María Cinta Subirats-Aleixandri, Las medidas cautelares: su instrumentalidad. Disponible en: http://www.derecho.com/articulos/2001/07/15/las-medidas-cautelares-su-instrumentalidad/ 
que el periculum in mora, el periculum in damni o in facti, esto es, un 'perjuicio irreparable', que se produciría si no se otorga (total o parcialmente) alguna prestación al actor o peticionario. De ahí que en la cautelar satisfactiva o anticipatoria lo fundamental no es el periculum in mora sino periculum pranse (actual) o in futuro (eventual); procurando aventar el 'perjuicio irreparable', denominado periculum in damni, o sea, el peligro que involucra el hecho. Desde una perspectiva del efecto es conjuratoria y asistencial".

\section{ANTECEDENTES DE LAS MEDIDAS CAUTELARES ANTICIPATORIAS E INNOMINADAS EN EL PROCESO ARBITRAL INTERNACIONAL}

\section{A. Antecedentes de las medidas cautelares anticipatorias en el arbitraje internacional}

Resulta de gran importancia para el desarrollo de esta investigación estudiar los antecedentes de legislaciones y reglamentos de instituciones de arbitraje que prevén esta figura en el proceso arbitral, pues en este estudio del derecho comparado se pueden analizar los aspectos críticos del arbitraje en Colombia, desde la perspectiva de un arbitraje de avanzada que permite tomar aquellos mecanismos que pueden servir para dotar el arbitraje colombiano de un proceso arbitral efectivo.

Algunas instituciones han puesto al servicio del arbitraje procesos para la adopción de estas medidas cautelares anticipadas a las que se viene haciendo referencia, y que se dan en muchos casos antes de que el tribunal se haya constituido, como en los casos que se presentarán posteriormente.

Para esta investigación se citarán los siguientes ejemplos:

9 Giovanni Sartori, El debido concepto de lo cautelar. Disponible en: http://www.academia dederecho.org/upload/biblio/contenidos/SARTORI.pdf, http://www.academiadederecho.org/ upload/biblio/contenidos/SARTORI 


\section{Asociación de arbitraje francesa}

En Francia, para solicitar este tipo de medidas de carácter urgente existe el llamado juez réfere, que es un órgano especial que actúa en casos de necesidad, estos jueces de emergencia no están facultados para resolver el fondo del asunto sino que actúan únicamente cuando el órgano encargado de conocer de la cuestión principal no esté en cuestiones de hacerlo ${ }^{10}$, como regla general no cabe la adopción de estas medidas a inaudita altera parte, debido a que son procesos de extrema urgencia y las partes deben ser oídas, no obstante de forma excepcional y si concurren circunstancias especiales que lo exijan, se permitirá su adopción ex parte ${ }^{11}$.

\section{Referee prearbitral de la CCI}

La Cámara de Comercio Internacional, CCI, de París ha elaborado el reglamento Rules for a Pre-arbitral Referee Procedure. Por medio de este mecanismo, se permite la adopción de una medida cautelar de forma rápida por un tercero imparcial denominado referee, cuando el tribunal arbitral todavía no se ha constituido; para acceder a este mecanismo, es necesario que las partes lo hayan expresado en el acuerdo arbitral o en otro documento por separado; por lo general, el referee es nombrado por las partes de común acuerdo o por el presidente del tribunal arbitral ${ }^{12}$.

El referee está legitimado para adoptar cualquier medida cautelar en caso de urgencia ya sea para prevenir un daño inmediato o una pérdida irreparable, o para salvaguardar los derechos de alguna de las partes en el proceso, cuando el tribunal arbitral todavía no se ha formado; así mismo, se le atribuye competencia para ordenar

10 Francia, Cour de cassation, asunto Société d'exploitation du Cinema Rex v. Société Rex, 7 de junio de 1979.

11 Artículos 812 y 875 del Nouveau Code de Procédure Civile. Francia, Loi 2007-1787, Nouveau Code de Procédure Civile, 20 décembre 2007. Disponible en: https://www.legifrance.gouv.fr/ affichCode.do?cidTexte=LEGITEXT000006070716

12 Artículo 4, párr. 1 y 2 del Reglamento del referee prearbitral de la CCI. Cámara de Comercio Internacional, CCI, Reglamento de Arbitraje de la Cámara de Comercio Internacional, CCI (2012). Disponible en: http://www.sice.oas.org/dispute/comarb/icc/arbruls.asp, http://www. iccwbo.org/Data/Documents/Buisness-Services/Dispute-Resolution-Services/Mediation/ Rules/2012-Arbitration-Rules-and-2014-Mediation-Rules-SPANISH-version/ 
el pago de una de las partes y para acordar cualquier medida necesaria con el objeto de asegurar la futura realización de pruebas.

\section{Optional Rules for Emergency Measures of Protection, American Arbitration Association, AAA}

La Asociación Americana de Arbitraje, AAA, ha creado un mecanismo similar al de la CCI, llamado Optional Rules for Emergency Measures of Protection, proceso que pretende resolver las controversias dentro del marco arbitral. La AAA ha designado un grupo de árbitros de emergencia para que puedan actuar de inmediato en casos de extrema necesidad; la aplicación de esta norma depende de que las partes lo hayan pactado expresamente, ya sea en la cláusula arbitral o en acuerdo por separado y por otro lado, que se trate ciertamente de una emergencia.

El funcionamiento del proceso es sencillo: la parte interesada en obtener una medida cautelar anticipada debe notificarlo por escrito a la AAA y a la otra parte, especificando qué tipo de medida solicita y los motivos en los que basa su petición ${ }^{13}$.

Una vez recibida la solicitud, la AAA nombra a un árbitro en el plazo de un día; si el árbitro es recusado, deberá notificarse y se realizará un reemplazo en el término de un día contado a partir del momento en que el nombramiento sea conocido por las partes; posteriormente, el árbitro dará audiencia a las partes en el máximo de dos días a partir de su designación.

\section{Emergency Relief Rules, Organización Mundial de la Propiedad Intelectual, OMPI}

En la Organización Mundial de la Propiedad Intelectual se crearon las normas denominadas Emergency Relief Rules. La creación de estos mecanismos obedece a que en ocasiones se producían retrasos en la constitución del tribunal arbitral, por el tiempo necesario para su constitución o porque las partes deseaban paralizar este

13 Artículo 0-1 de las Optional Rules For Emergency Measures of Protection, de la AAA. Estados Unidos, Asociación Americana de Arbitraje, AAA, Reglamento de arbitraje internacional de la Asociación Americana de Arbitraje, AAA (1997). Texto disponible en español e inglés: http:// www.intracen.org/Reglamento-de-Arbitraje-Internacional-de-la-Asociacion-Americana-deArbitraje-1997/, http://www.intracen.org/International-Arbitration-Rules-of-the-AmericanArbitration-Association-2001/ 
procedimiento por la ventaja que esa parálisis les podía reportar ${ }^{14}$. Precisamente, esta última circunstancia condujo a la formulación de las normas de emergencia en materia cautelar anticipatoria.

Estas medidas se pueden ordenar tanto en presencia de ambas partes como inaudita altera parte, aunque esta última opción queda supeditada a circunstancias excepcionales; además, los árbitros gozan de poderes muy amplios, en el sentido de estar legitimados para adoptar cualquier tipo de medida que consideren conveniente; en ambos casos, sea inaudita parte o con presencia de la otra parte, previa solicitud de una parte, el centro de arbitraje procederá a constituir el panel de árbitros en un plazo máximo de 24 horas $^{15}$. No obstante, si las partes nombraron a quien actuaría de árbitro de emergencia, será este quien se designe ${ }^{16}$. A falta de previsión de las partes, el centro será el encargado de hacer el nombramiento ${ }^{17}$. En caso de recusación de algunos de sus miembros, se procederá a su sustitución en un plazo no superior a 24 horas $^{18}$.

\section{Associazione Italiana per l'Arbitrato}

\section{La Asociación Italiana para el Arbitraje también prevé la figura de} estas medidas cautelares anticipadas o prearbitrales; la competencia se atribuye en este caso al comité permanente ${ }^{19}$. El artículo 17 párrafo 5 dispone: "si el tribunal arbitral todavía no se ha consti-

14 Richard Allan Horning, Interim Measures of Protection; Security for Claims and Costs, and Commentary on the WIPO Emergency Relief Rules, 9 American Review of International Arbitration, 1-4 (1998).

15 Artículo VI A, Emergency Relief Rules. Organización Mundial de la Propiedad Intelectual, OMPI, Reglamento de Arbitraje del Tribunal de la Organización Mundial de la Propiedad Intelectual, OMPI. Disponible en: http://www.wipo.int/amc/es/arbitration/rules/, http://www. wipo.int/amc/en/arbitration/rules/

16 Artículo VII A, Emergency Relief Rules. Organización Mundial de la Propiedad Intelectual, OMPI, Reglamento de Arbitraje del Tribunal de la Organización Mundial de la Propiedad Intelectual, OMPI. Disponible en: http://www.wipo.int/amc/es/arbitration/rules/, http://www. wipo.int/amc/en/arbitration/rules/

17 Artículo VII B, Emergency Relief Rules. Organización Mundial de la Propiedad Intelectual, OMPI, Reglamento de Arbitraje del Tribunal de la Organización Mundial de la Propiedad Intelectual, OMPI. Disponible en: http://www.wipo.int/amc/es/arbitration/rules/, http://www. wipo.int/amc/en/arbitration/rules/

18 Artículo VIII, Emergency Relief of Rules. Organización Mundial de la Propiedad Intelectual, OMPI, Reglamento de Arbitraje del Tribunal de la Organización Mundial de la Propiedad Intelectual, OMPI. Disponible en: http://www.wipo.int/amc/es/arbitration/rules/, http://www. wipo.int/amc/en/arbitration/rules/

19 Artículo 17, párr. 5. Italia, Associazione Italiana per l’Arbitrato, AIA, Asociación Italiana para Arbitraje, Reglamento de la Asociación Italiana para Arbitraje. Disponible en: http:// arbitratoaia.com/images/immagini/Regolamento_Arbitrato_AIA_2016.pdf 
tuido, las medidas cautelares pueden ser adoptadas por el comité permanente y podrán ser revocadas o modificadas en cualquier momento por el comité y después de la constitución del tribunal arbitral, por este mismo".

El funcionamiento y finalidad de estas normas son idénticos a los mencionados ya anteriormente.

\section{Centro Empresarial de Conciliación y Arbitraje, CEDCA}

El reglamento del CEDCA permite la adopción de medidas cautelares anticipadas. Este interesante mecanismo se alinea sin duda con el carácter urgente propio de las medidas cautelares pues permite su adopción inmediata, apenas la solicitud de arbitraje es presentada, con lo cual se acrecienta la posibilidad de proteger provisional y rápidamente el derecho en litigio sin esperar la realización de todos los procedimientos para la constitución del tribunal arbitral que decidirá de fondo sobre el asunto.

Además, el artículo 35.2 del reglamento del CEDCA dispone que salvo acuerdo en contrario de las partes, cuando circunstancias de urgencia lo ameriten, cualquiera de las partes podrá antes del nombramiento de los árbitros y previo el pago de los honorarios y pagos previstos en el apéndice I del reglamento, solicitar al directorio del CEDCA, que designe de la lista oficial de árbitros, un tribunal arbitral compuesto a juicio del director ejecutivo por uno o tres árbitros, para que resuelva exclusivamente sobre el decreto de las medidas cautelares solicitadas, la designación de estos árbitros la hará el directorio del CEDCA de manera rotativa entre los inscritos en la lista oficial de árbitros que no estén actuando en ese momento como tales en un arbitraje administrado por el CEDCA ${ }^{20}$.

20 Artículo 35.2, CEDCA. Venezuela, Centro Empresarial de Conciliación y Arbitraje, CEDCA, Reglamento de Conciliación y Arbitraje. Disponible en: http://www.cedca.org.ve/documentos/ normativa_arbitraje/iii.\%20Reglamento\%20CEDCA.pdf 


\section{B. Antecedentes de las medidas cautelares innominadas en el proceso arbitral internacional}

Numerosos instrumentos internacionales dan una discrecionalidad absoluta a los árbitros para dictar la medida cautelar que consideren necesaria para la protección del derecho en litigio; las medidas cautelares innominadas dan un poder cautelar general al árbitro con el fin de que pueda asegurar sin limitaciones, la efectividad y asegurar el resultado procesal de lo que aspira la parte. Para esta investigación, se estudiarán las diferentes normativas que ofrecen la aplicación de estas medidas cautelares innominadas tanto en las legislaciones como en los reglamentos de las instituciones de arbitraje, de la siguiente manera:

\section{Las medidas cautelares innominadas en España}

La Ley de Enjuiciamiento Civil española consagra en su artículo 23 que "salvo acuerdo en contrario de las partes, los árbitros podrán, a instancia de ellas, adoptar las medidas cautelares que estimen necesarias respecto del objeto en litigio..."21.

\section{Las medidas cautelares innominadas en Inglaterra y Suecia}

La Ley de Arbitraje inglesa de 1996 y la sueca de 1999 consagran una normativa similar "salvo que las partes hayan acordado lo contrario, a solicitud de una parte, los árbitros pueden decidir que durante el trámite arbitral, la parte contraria debe asumir ciertas medidas cautelares para asegurar la pretensión objeto del arbitraje..."22.

21 España, Ley 1/2000, 7 de enero, Ley de Enjuiciamiento Civil, artículo 23. Disponible en: https://boe.es/buscar/act.php?id=BOE-A-2000-323

22 Inglaterra, Ley de Arbitraje Inglesa de 1996. Disponible en: http://www.legislation.gov.uk/ ukpga/1996/23/contents. Suecia, Ley de Arbitraje, 4 de marzo de 1999. Disponible en: http:// www.sc.ehu.es/dpwlonaa/legislacion/ARBITRAJE\%20INTERNACIONAL/Ley\%20Sueca/ Ley\%20sueca.htm, http://www.uni-kiel.de/leobalt/Datenbank/Schweden/The $\% 20$ Swedish $\% 20$ Arbitration $\% 20$ Act $\% 20$ of $\% 201999$.htm 


\section{Reglamento de Arbitraje de la CCI (Cámara de Comercio Internacional)}

El reglamento de la Corte Internacional de Arbitraje de la Cámara de Comercio Internacional, en su artículo 23-1 establece lo siguiente, "Medidas cautelares, 1). A menos que se haya acordado de otro modo por las partes, el tribunal arbitral puede, tras el envío de la documentación a petición de una de las partes, ordenar cualquier medida cautelar que considere apropiada. Su adopción se puede condicionar a la constitución de garantías adecuadas. Las medidas aludidas en el presente artículo son adoptadas mediante resolución motivada o si es necesario bajo la forma de sentencia, si el tribunal arbitral lo considera adecuado"23.

\section{Reglamento de Arbitraje de la American Arbitration Association, AAA}

El reglamento de la Asociación Americana de Arbitraje (AAA) también establece en su artículo 21, lo siguiente, "Medidas Provisionales de Protección, 1). A solicitud de cualquier parte, el tribunal podrá tomar aquellas medidas provisionales necesarias, incluyendo mandamiento de hacer o no hacer y medidas para la protección o conservación de propiedad"24.

\section{Reglamento del Tribunal Arbitral de la Organización Mundial de la Propiedad Intelectual, OMPI}

El reglamento de arbitraje de la Organización Mundial de la Propiedad Intelectual (WIPO, por sus siglas en inglés) señala en su artículo 48, "Medidas provisionales o conservatorias y garantía para las demandas y costas, a) A petición de cualquiera de las partes, el Tribunal podrá dictar cualquier orden provisional o tomar otras

23 Cámara de Comercio Internacional, CCI, Reglamento de Arbitraje de la Cámara de Comercio Internacional, CCI (2012). Disponible en: http://www.sice.oas.org/dispute/comarb/icc/arbruls. asp, http://www.iccwbo.org/Data/Documents/Buisness-Services/Dispute-Resolution-Services/Mediation/Rules/2012-Arbitration-Rules-and-2014-Mediation-Rules-SPANISH-version/

24 Asociación Americana de Arbitraje, AAA, Reglamento de arbitraje internacional de la Asociación Americana de Arbitraje, AAA (1997). Texto disponible en español e inglés: http:// www.intracen.org/Reglamento-de-Arbitraje-Internacional-de-la-Asociacion-Americanade-Arbitraje-1997/, http://www.intracen.org/International-Arbitration-Rules-of-the-American-Arbitration-Association-2001/ 
medidas provisionales que estime necesarias respecto del objeto de la controversia, incluidas las medidas cautelares, así como otras destinadas a la conservación de los bienes que constituyan el objeto de la controversia, tales como ordenar que los bienes se depositen en manos de un tercero o que se vendan los bienes perecederos. El Tribunal podrá supeditar la concesión de dichas medidas a una garantía apropiada proporcionada por la parte peticionaria"25.

\section{Reglamento de arbitraje de la London Court of International Arbitration, LCIA}

El reglamento de la Corte de Arbitraje Internacional de Londres, estipula lo siguiente, "Artículo 25. Medidas provisionales de protección y de aseguramiento de bienes litigiosos. Salvo acuerdo por escrito en contrario de las partes, el Tribunal Arbitral, a solicitud de una de las partes, estará facultado para: a) ordenar a cualquier demandada o demandada reconvencional que afiance parcial o totalmente la cuantía reclamada, sea por medio de depósito, aval bancario o de cualquier otro modo y manera que el Tribunal Arbitral estime oportuno"26.

\section{LAS MEDIDAS CAUTELARES ANTICIPATORIAS EN EL PROCESO ARBITRAL EN COLOMBIA}

En Colombia, hay diferentes instituciones de orden procesal, como la jurisdicción y la competencia, que además constituyen presupuestos procesales, como es conocido por la comunidad académica que estudia lo concerniente al caso, por regla general. Sin embargo, en aplicación de lo establecido en el artículo 116 de la Constitución Política ${ }^{27}$, excepcionalmente y de forma transitoria también podrán

25 Organización Mundial de la Propiedad Intelectual, OMPI, Reglamento de Arbitraje del Tribunal de la Organización Mundial de la Propiedad Intelectual, OMPI. Disponible en: http:// www.wipo.int/amc/es/arbitration/rules/, http://www.wipo.int/amc/en/arbitration/rules/

26 Inglaterra, Corte de Arbitraje Internacional de Londres, LCIA, Reglamento de la Corte (1998). Disponible en: http://www.lcia.org/Dispute_Resolution_Services/LCIA_Arbitration_Rules. aspx

27 Colombia, Constitución Política, 7 de julio de 1991. Versión corregida, 116 Gaceta Constitucional, 20 de julio de 1991, disponible en: http://www.secretariasenado.gov.co/senado/basedoc/ constitucion_politica_1991.html 
administrar justicia los particulares cuando actúen como conciliadores, jurados o árbitros.

Lo anterior implica que el arbitraje que constituye un mecanismo alternativo al proceso, tal como establece la Ley 1285 de $2009^{28}$, se haya convertido en uno de los que implican la actuación excepcional de los particulares que para los casos en que sean habilitados por las partes, administren lo que por regla general se concibe como la justicia en cabeza de los jueces delegados por el mismo Estado para realizar esta función.

Aunque el arbitraje con el tiempo se fue convirtiendo en el país en un mecanismo que se caracterizaba por ser la elección de las grandes empresas y multinacionales con presencia en Colombia por su celeridad y el alto nivel de experticia de quienes actuaban como árbitros, no todo eran aspectos positivos pues históricamente en el país este mecanismo estaba regulado por un gran número de decretos que terminaban haciendo su desarrollo algo caótico, por lo que se pensó en la expedición de un nuevo ordenamiento jurídico que unificara lo concerniente a la regulación del arbitraje nacional y que respondiera a las tendencias que en esta materia han sido consecuencias mismas de fenómenos como la globalización y el comercio entre diferentes países del mundo. Como ejemplo se puede pensar en los contratos de compra y venta de mercaderías internacionales en que por regla general se puede ver cómo las empresas que realizan este tipo de operaciones de forma expresa estipulan que en caso de conflictos generados a partir del contrato, deberán ser árbitros los que decidan la suerte del mismo.

Por todo lo anterior, en Colombia fue expedida la Ley 1563 de 2012, vigente actualmente, y que se denomina Estatuto Arbitral Colombiano. De otro lado, el Código General del Proceso implementa la nueva figura de las medidas cautelares innominadas, que podrán decretarse por el juez pero también por un árbitro, lo cual genera la siguiente conclusión en el ámbito nacional: las medidas cautelares pueden ser anticipatorias o dentro del proceso y estas, a su vez, pueden ser nominadas e innominadas.

28 Colombia, Ley 1285 de 2009, por medio de la cual se reforma la Ley 270 de 1996 Estatutaria de la Administración de Justicia, 47.240 Diario Oficial, 22 de enero de 2009. Disponible en: http://www.secretariasenado.gov.co/senado/basedoc/ley_0446_1998.html 
En cuanto a las medidas cautelares anticipatorias - es decir, aquellas que se dan antes de que el demandado en un proceso arbitral sea notificado de que existe una demanda en su contra-, hay un vacío normativo, pues hasta el momento, el Estatuto Arbitral en Colombia solo presenta un esquema según el cual la solicitud de medidas se hace ante el Tribunal de Arbitraje, ya instalado, y deja de lado figuras que en el ámbito internacional son utilizadas — por ejemplo, por la Cámara de Comercio Internacional_, como los árbitros de urgencia.

Es decir, las medidas cautelares preventivas, anticipadas o prearbitrales son un instrumento que se presenta a la parte demandada antes del inicio de las actuaciones arbitrales, para asegurar sus derechos ante el temor fundado del incumplimiento de su contraparte. En el arbitraje, las medidas cautelares pueden solicitarse antes de la constitución del tribunal arbitral o, incluso, antes del inicio de las actuaciones arbitrales (las llamadas medidas cautelares anticipadas) o bien una vez se haya constituido el tribunal arbitral ${ }^{29}$.

Ahora bien, en Colombia, hay consenso acerca de que los árbitros por administrar justicia de forma transitoria, no podrían decretar este tipo de medidas sin que se instale el respectivo tribunal; por esta razón, se puede pensar que implementar una figura como la que se menciona anteriormente en principio iría contra el artículo 116 de la Constitución Política, porque básicamente se estaría diciendo con esto que habrá unos árbitros que sí administran justicia de forma permanente.

Lo que sí se puede decir es que hay vacíos normativos que pueden generar problemas prácticos en el futuro. Esta investigación pretende abordarlos y, de manera descriptiva pero también haciendo un análisis integral de las normas que regulan el tema, presentar respuestas a la comunidad académica de la siguiente manera:

29 Pedro Perera \& Humberto Angrisano, Las medidas cautelares en el arbitraje, Revista Comité de Arbitraje, 3 (2013). Disponible en: http://www.venamcham.org/index.php?option=com_ content\&id=1296\%3Acomite-de-arbitraje-las-medidas-cautelares-en-el-arbitraje-febrero2013\&Itemid $=19$ 


\section{A. Aspectos críticos}

Como se puede evidenciar, aunque las medidas cautelares como institución no configuran una novedad, esta investigación pretende responder los siguientes interrogantes: ¿Cuáles son las características de las medidas cautelares innominadas en el proceso arbitral en Colombia? ¿Cuáles son las características de las medidas cautelares anticipatorias en el proceso arbitral en Colombia? ¿Cuál es la situación actual respecto a las medidas cautelares anticipatorias en el proceso arbitral en Colombia? ¿Cuál es la situación de las medidas cautelares innominadas en el proceso arbitral en Colombia? ¿Qué teoría y referentes legales actuales y pertinentes favorecen la solicitud, decreto y práctica de las medidas cautelares anticipatorias en el proceso arbitral en Colombia? ¿Qué teoría y referentes legales actuales y pertinentes favorecen la solicitud, decreto y práctica de las medidas cautelares innominadas en el proceso arbitral en Colombia? ¿Qué alternativas se pueden proponer en Colombia para la solicitud, decreto y práctica de las medidas cautelares anticipatorias en el proceso arbitral en Colombia? ¿Qué alternativas se pueden proponer en Colombia para la solicitud, decreto y práctica de las medidas cautelares innominadas en el proceso arbitral en Colombia?

Por ello se desarrollan todos estos aspectos de la siguiente manera:

\section{Solicitud}

En primer lugar, la Ley 1563 de 2012 no regula el procedimiento para la solicitud de medidas cautelares anticipadas sino que se limita a su tratamiento cuando se dan dentro del proceso arbitral. Por ello, resulta de gran importancia abordar esta figura y ofrecer una regulación apropiada, para que por medio del arbitraje también se garantice la efectividad de los derechos reconocidos por la Ley sustancial, con el fin de garantizar el derecho a la tutela judicial efectiva.

Una de las características especiales de las medidas cautelares anticipatorias es que se surten antes de notificado el demandado del proceso en su contra, dicho sea, para garantizar la efectividad 
del objeto del proceso $\mathrm{o}^{30}$. Sin embargo, en el proceso arbitral, hay un problema que impide la realización de este tipo de medidas y se expondrá a continuación.

Para evaluar este punto, deben separarse tres momentos, que darán mayor claridad sobre el problema presente: i) el momento de la solicitud de las medidas cautelares en el proceso arbitral; ii) el momento en que el tribunal adquiere competencia para el conocimiento y decreto de medidas cautelares; y iii) el momento de la notificación al demandado en el proceso arbitral.

1. El momento de la solicitud: según el Estatuto Nacional e Internacional de Arbitraje (en adelante, ENAI), Ley 1563 de 2012, en su artículo 32, la solicitud de medidas cautelares en los procesos arbitrales se hará: "A petición de cualquiera de las partes" y ante lo cual "el tribunal podrá ordenar las medidas cautelares que serían procedentes de tramitarse el proceso ante la justicia ordinaria o la contencioso administrativa". Dado que el ENAI enuncia que la petición o solicitud de las medidas cautelares, las hará el accionante ante el tribunal de arbitraje, se deduce que este —el tribunal—ya debe estar instalado al momento de realizarse la solicitud de medidas, es decir, el momento de la solicitud, es posterior a la instalación ${ }^{31}$.

2. El momento del conocimiento: si bien es cierto, el proceso arbitral inicia desde el momento mismo de la presentación de la demanda ante el tribunal de arbitraje, como lo enuncia el artículo 12 del ENAI "Iniciación del proceso arbitral. El proceso arbitral comenzará con la presentación de la demanda". No es sino hasta la instalación del tribunal de arbitramento, cuando este adquiere facultades jurisdiccionales y puede utilizar sus poderes de instrucción para resolver el proceso; en la instalación del tribunal de arbitraje según el artículo 20 del ENAI se presentará la demanda, y se estudiará "la admisión, la inadmisión y el rechazo de la demanda". Es decir, el tribunal de arbitraje no conoce de la litis, sino hasta el momento mismo de la audiencia de instalación.

30 Piero Calamandrei, Introducción al estudio sistemático de las providencias cautelares, 45 (Editorial Bibliográfica Argentina, Buenos Aires, 1984).

31 Colombia, Ley 1563 de 2012, por medio de la cual se expide el Estatuto de Arbitraje Nacional e Internacional y se dictan otras disposiciones, Artículo 20, 48.489 Diario Oficial, 12 de julio de 2012. Disponible en: http://www.secretariasenado.gov.co/senado/basedoc/ley_1563_2012. html 
3. El momento de notificación de la demanda: en el proceso arbitral, si bien la parte se entera de que entabla el proceso desde el instante en que es requerida para la composición de los árbitros (salvo estipulación en contrario en el pacto arbitral), es justo en el momento de la instalación del tribunal cuando en audiencia el demandado es notificado. Por ello, se puede concluir que en la instalación del tribunal se surte efecto la notificación del auto admisorio de la demanda (lo cual se asemeja a la terminología del proceso civil).

Dado que en la instalación está presente la contraparte (si bien hay término de traslado de la demanda) conociendo de la litis que se entabla contra ella, entonces cabe cuestionar: ¿Cómo se debe hacer el tratamiento de las medidas cautelares anticipatorias en el proceso arbitral en Colombia?

Se pueden evidenciar entonces dos momentos procesales sobre los cuales versa este dilema: i) antes de instalado el tribunal, cuando el accionante requiere el decreto de la medida cautelar anticipatoria, pero por carecer de competencia (aún) el tribunal arbitral para revisar del proceso, no puede ser decretada; y ii) después de instalado el tribunal, cuando al adquirir competencia este ya puede decretar las medidas cautelares solicitadas, pero la contraparte ya ha sido notificada también del proceso en su contra y, por tanto, carece de utilidad la medida cautelar cuyo fin sea poseer carácter anticipatorio.

Para contextualizar lo anterior, es necesario plantear el siguiente ejemplo hipotético: una empresa que celebra un contrato de compraventa de mercaderías con otra empresa y que en el mismo consagra cláusula compromisoria habilitando el arbitraje en caso de un conflicto originado en ese negocio jurídico desea solicitar que se decrete una medida cautelar en el proceso arbitral sin que se notifique a la empresa demandada para garantizar la efectividad de un derecho que considera será objeto de litigio. Sin embargo, nada prevé al respecto el Estatuto Arbitral en Colombia. ¿Se podría decretar la medida cautelar anticipada en este caso? Y de ser positiva la respuesta, ¿ante quién debería solicitarse? ¿Quién debería decretarla? y ¿cuál debería ser el procedimiento para hacerlo? En caso de ser negativa la respuesta, ¿cómo se garantizaría un derecho que requiere este tipo de medida en el proceso arbitral en Colombia? 
Es decir, sin importar si la respuesta al primer interrogante planteado es positiva o negativa, esta investigación - a partir de una propuesta que adopta diferentes criterios desarrollados por el arbitraje internacional, y el estudio sistemático de la Ley 1563 de 2012, la Ley 1564 de 2012 (Código General del Proceso) y la Constitución Política - abordará las posibles soluciones que frente a esta problemática podrían adoptarse en el país.

\section{Decreto}

Entendiendo las vicisitudes que conlleva la solicitud de las medidas cautelares en los procesos arbitrales, cuyo carácter anticipatorio es normativamente imposible, entonces se debe ahora abordar el decreto de estas medias que, en principio (y se verá más adelante otro vacío jurídico), las decretará siempre el tribunal.

El ENAI establece que para el proceso de decreto de medidas cautelares "se someterán a las normas del Código de Procedimiento Civil, el Código de Procedimiento Administrativo y de lo Contencioso Administrativo [CPACA] y a las disposiciones especiales pertinentes" siendo la regencia del CPACA solo posible en casos donde "sea parte una entidad pública o quien desempeñe funciones administrativas".

Las medidas que podrá decretar el tribunal arbitral son tanto las nominadas de los códigos referidos, como "cualquier otra medida cautelar que encuentre razonable para la protección del derecho objeto del litigio, impedir su infracción o evitar las consecuencias derivadas de la misma, prevenir daños, hacer cesar los que se hubieren causado o asegurar la efectividad de la pretensión".

Los criterios del decreto de medidas cautelares en estos procesos son básicamente los establecidos de manera reiterada por la doctrina ${ }^{32}$ : i) "la legitimación o interés para actuar de las partes"; ii) "la existencia de la amenaza o la vulneración del derecho" lo que la doctrina expone básicamente como el periculum in mora; iii) "la apariencia de buen derecho" o también denominado fumus boni iuris; y iv) "la necesidad, efectividad y proporcionalidad de la medida".

32 Jordi Nieva-Fenoll, El elemento psicológico en la adopción de las medidas cautelares, en Memorias del IX Congreso Internacional de Derecho Procesal, San José de Cúcuta, 2 y 3 de agosto de 2013, 107-128 (Universidad Libre Seccional Cúcuta, Cúcuta, 2013). Disponible en: http:// portales.te.gob.mx/seminario/sites/portales.te.gob.mx.seminario/files/materiales/Jordi $\% 20$ Nieva.pdf 
Teniendo el tribunal el criterio de establecer su alcance, duración, modificación, sustitución o cese; y, por tanto, pudiendo otorgarlas ultra, extra o minus petita al solicitante.

Uno de los puntos novedosos que establece la vigencia del ENAI es que en la medida en que protege el objeto del proceso, al asegurar el cumplimiento de las pretensiones, dispone que las medidas "también podrán tener como objeto recaudar elementos de prueba que pudiesen ser relevantes y pertinentes para la controversia" ${ }^{33}$ ante lo cual resulta en una posición ventajosa en la garantía y efectividad de los derechos. Sin embargo, si con el decreto de la medida se busca asegurar material probatorio en manos de la contraparte, el recaudo de elementos de prueba tendría mayor efectividad si se aplicara en un momento previo a la notificación al demandado (medida anticipatoria).

Hay un requisito añadido por el ENAI para que sea decretada cualquiera de las anteriores medidas cautelares innominadas, ante lo cual "el demandante deberá prestar caución equivalente al veinte por ciento (20\%) del valor de las pretensiones estimadas en la demanda, para responder por las costas y perjuicios derivados de su práctica". Sin embargo, el tribunal, de oficio o a petición de parte, podrá aumentar o disminuir el monto de la caución cuando lo considere razonable, o fijar uno superior al momento de decretar la medida ${ }^{34}$.

Otro punto controversial podría considerarse un eventual vacío jurídico, que permitiría el decreto de medidas cautelares previas a la iniciación del proceso arbitral, con base en la distinción de dos momentos: el primero desde la presentación de la demanda, así exista pacto arbitral, el juez no puede rechazar de plano, ni inadmitir el trámite de la misma, dado el parágrafo contenido en el artículo 90 del Código General del Proceso, CGP. Sin embargo, por tratarse de un proceso ante la jurisdicción ordinaria, el demandante sí podría solicitar desde que acciona el aparato jurisdiccional, que se

33 Colombia, Ley 1563 de 2012, por medio de la cual se expide el Estatuto de Arbitraje Nacional e Internacional y se dictan otras disposiciones, artículo 32, parágrafo, 48.489 Diario Oficial, 12 de julio de 2012. Disponible en: http://www.secretariasenado.gov.co/senado/basedoc/ ley_1563_2012.html. Cabe recalcar que este tipo de medidas el mismo parágrafo las extiende a cualquier operador jurisdiccional.

34 Colombia, Ley 1563 de 2012, por medio de la cual se expide el Estatuto de Arbitraje Nacional e Internacional y se dictan otras disposiciones, artículo 32, parágrafo, 48.489 Diario Oficial, 12 de julio de 2012. Disponible en: http://www.secretariasenado.gov.co/senado/basedoc/ ley_1563_2012.html 
decrete una medida cautelar anticipatoria, la cual surtiría efecto antes o justo notificado el demandado del proceso en su contra. El segundo momento se daría luego de la notificación del demandado, cuando al correrse el traslado él tendría la oportunidad de alegar la existencia del pacto arbitral, ante lo cual - como lo estipula el mismo artículo del CGP — se "provocará la terminación del proceso cuando se declare probada la excepción previa respectiva" siendo así que: i) ya estaría en vigencia la medida cautelar decretada; y ii) perdería competencia el juez que la decretó, ante lo cual no podría desmontarla, modificarla o sustituirla, sino el ahora competente tribunal del arbitraje.

\section{Práctica}

En cuanto a la práctica y al levantamiento de las medidas cautelares, se asemeja mucho al normalmente acaecido en la jurisdicción ordinaria, teniendo como factor controversial la fuerza de cumplimiento que tienen las emanaciones de los tribunales arbitrales, pero que se resuelve mediante la práctica de condicionamientos. Hay en el ENAI una salvedad en cuanto a las medidas cautelares que permite "impedir su práctica o solicitar su levantamiento o modificación", y es cuando se trata de pretensiones pecuniarias ante lo cual basta la prestación de una caución por parte del demandado, con el fin de garantizar el cumplimiento de la sentencia o de prestar indemnización en caso de la imposibilidad para cumplirla. No podrá prestarse caución cuando las medidas cautelares no estén relacionadas con pretensiones económicas o procuren anticipar materialmente el fallo. Entiéndase en este punto la voluntad que une a las partes al proceso, y la tutela judicial efectiva garantizada mediante el desarrollo de las medidas cautelares en especial las anticipatorias $^{35}$.

En cuanto a la fuerza de cumplimiento que poseen los tribunales arbitrales, puede decirse que este problema se resuelve a partir de dos aspectos: i) en principio, las partes por voluntad propia se

35 Luiz Guilherme Marinoni, La evolución de la técnica anticipatoria y de la tutela preventiva en Brasil, en Memorias del XXXIV Congreso Colombiano de Derecho Procesal, Medellín, 11, 12 y 13 de septiembre de 2013, 975-993 (Universidad Pontificia Bolivariana, Medellín, 2013). Disponible en: https://docs.google.com/viewer?a=v\&pid=sites\&srcid=ZGVmYXVsdGRvbW Fpbnxjb25ncmVzb2RjaG9wcm9jZXNhbHxneDo2NmNkM2Q1MGUwMWZhODAy 
dispusieron a la competencia de un tribunal arbitral, así que ha de suponerse que ellas actuarán en favor del cumplimiento de las órdenes del tribunal; ii) ahora en caso de no ser así, los tribunales arbitrales disponen de la posibilidad de comisionar a los jueces civiles (o administrativos si alguna de las partes es entidad pública o cumple funciones públicas) para que estos practiquen o ejecuten las medidas adoptadas por el tribunal, así lo refiere el artículo 32 del ENAI.

\section{B. Propuesta}

En respuesta a la problemática planteada frente a la solicitud, decreto y práctica de las medidas cautelares anticipatorias e innominadas en el proceso arbitral en Colombia, se presenta la siguiente propuesta como resultado de la investigación:

\section{Medidas cautelares anticipatorias en el proceso arbitral en Colombia}

Teniendo en cuenta que hay un vacío normativo frente a las medidas cautelares anticipatorias en el proceso arbitral en Colombia, es necesario plantear las siguientes opciones:

\section{a. Solicitud}

El primer interrogante que se propone responder esta investigación es precisamente frente a la solicitud de este tipo de medidas, para lo que se presentan las siguientes propuestas:

La solicitud de la medida cautelar anticipatoria en el proceso arbitral debería hacerse:

1. A partir de un proceso prearbitral, en que la parte interesada haga la respectiva solicitud al Centro de Arbitraje y que un número impar de árbitros conforme un Tribunal de Urgencia que tenga competencia para decretar la misma a elección de las partes o, en caso de no ser así, por el Centro designado por estas.

2. A partir de la implementación de la figura de árbitros de urgencia designados por los respectivos Centros de Arbitraje para decretar estas medidas, sin necesidad de un proceso prearbitral, lo 
que en principio se puede pensar iría en contra de la Constitución Política, entendiendo que los particulares, en calidad de árbitros solo pueden administrar justicia de forma transitoria y no permanente.

3. A partir de la aplicación del apoyo concurrente que debe haber entre los poderes y la armonización de lo que pueda entenderse debe darse en la administración de justicia, incluso a partir de la interpretación de la Ley 1563 de 2012, se pensaría que serían los jueces civiles del circuito los llamados a tener competencia frente a la solicitud de estas medidas cautelares anticipatorias en el proceso arbitral para una posterior ratificación de ellas por parte de los árbitros que vayan a conocer del conflicto, pero esto iría en contra del principio de autonomía de voluntad de partes y el principio de habilitación que se predican en materia de arbitraje nacional e internacional.

Sin embargo, el parágrafo del artículo 32 de la Ley 1563 de 2012, haciendo referencia a las medidas que se pueden decretar dentro del proceso arbitral para recaudar elementos de prueba, que de por sí deben ser anticipatorias, establece que "quien ejerza funciones jurisdiccionales podrá decretar medidas cautelares para este propósito en los procesos sometidos a su conocimiento, sean o no procesos arbitrales", lo que al parecer fortalece el argumento anteriormente planteado de que sean los jueces civiles del circuito quienes puedan decretar este tipo de medidas, aunque no sean estos los habilitados por las partes.

\section{b. Decreto}

Respecto del interrogante planteado en la investigación frente al decreto de las medidas cautelares anticipatorias en el proceso arbitral en Colombia, se presentan las siguientes propuestas:

El decreto de la medida cautelar anticipatoria en el proceso arbitral debería hacerse:

1. A partir de un proceso arbitral como se explicó, en el aparte que trata de la solicitud de las mismas.

2. A partir de la implementación de la figura de unos árbitros de urgencia que estén designados de forma permanente por el Centro de Arbitraje para cumplir con esto. 
3. A partir de la competencia que se designe en los jueces civiles del circuito, con base en los argumentos anteriormente planteados.

\section{c. Práctica}

La práctica de la medida cautelar anticipatoria (nominadas e innominadas) en el proceso arbitral debería hacerse:

1. A partir de un proceso arbitral como se explicó, en el aparte que trata de la solicitud de las mismas, y antes de que el demandado tenga conocimiento de ellas, es decir, antes de que se instale en la forma en que lo concibe la Ley 1563 de 2012, el Tribunal Arbitral.

2. A partir de la implementación de la figura de unos árbitros de urgencia que estén designados de forma permanente por el Centro de Arbitraje para cumplir con esto, antes de que el demandado tenga conocimiento de las mismas, es decir, antes de que se instale en la forma en que lo concibe la Ley 1563 de 2012, el Tribunal Arbitral.

3. A partir de la competencia que se designe en los jueces civiles del circuito, con base en los argumentos anteriormente planteados, es decir, antes de que se instale en la forma en que lo concibe la Ley 1563 de 2012, el Tribunal Arbitral, pero en este caso la medida sería susceptible de ser revocada por los árbitros habilitados por las partes para conocer del conflicto, lo que en la práctica podría generar un perjuicio frente a la parte que se decretó la medida, pero esto podría mitigarse con una caución que respalde el decreto y práctica de la medida. 


\section{CONCLUSIONES}

Las medidas cautelares en el proceso arbitral en Colombia pueden ser anticipatorias o dentro del proceso arbitral (después de notificado el demandado). Esto implica que en el proceso arbitral deben implementarse figuras o herramientas ya sea directamente confiadas a otros particulares - como lo sería en la implementación de los árbitros de urgencia en Colombia, figura que existe en la Cámara de Comercio Internacional de París pero de la que nada se ha dicho internamente. De lo contrario, serían los jueces por medio del principio de habilitación y en el entendido de que el Código General del Proceso no establece que el pacto arbitral sea una causal de rechazo ni de inadmisión de la demanda sino que será la parte demandada la que deba alegar su existencia.

Las medidas cautelares anticipatorias en el proceso arbitral en Colombia (nominadas e innominadas) no presentan regulación expresa en la Ley 1563 de 2012. Esta situación evidentemente hace que en aplicación del principio de integración y de lo que el mismo Estatuto establece, es decir, que se remite a esta normativa, tenga aplicación el Código General del Proceso.

Las medidas cautelares anticipatorias (nominadas e innominadas) pueden ser decretadas por los árbitros en Colombia, en aplicación de la Ley 1563 de 2012 y el Código General del Proceso. Lo anterior, debido a que esta es una de las facultades jurisdiccionales que tienen los particulares cuando transitoria y excepcionalmente son administradores de justicia, en el desarrollo de este mecanismo alternativo al proceso.

En Colombia, es necesaria la adopción de una solución ante el vacío normativo frente a la solicitud, decreto y práctica de las medidas cautelares anticipatorias (nominadas e innominadas) planteado a partir de la investigación realizada.

La posibilidad que se les ofrece a las partes que acuden al proceso judicial para solicitar medidas cautelares también debe existir para aquellos que elijan el arbitraje como mecanismo alternativo al proceso para la solución de un litigio. 


\section{BIBLIOGRAFÍA}

\section{Libros}

Araque-Benzo, Luis Alfredo, Manual de arbitraje comercial (Fundación Editorial Jurídica Venezolana, Caracas, 2011).

Barrozo-Osorio, Tulia del Carmen, Eficacia de las medidas cautelares en los procesos declarativos ordinarios en la legislación colombiana (Organización Digital Casa Editorial S.A., Cartagena de Indias D.T.C - Colombia, 2008).

Bejarano-Guzmán, Ramiro, Código General del Proceso y Código de Procedimiento Civil comparativo (Editorial Externado de Colombia, Bogotá, 2012).

Bejarano-Guzmán, Ramiro, La jurisprudencia arbitral en Colombia, Tomo V (Editorial Externado de Colombia, Bogotá, 2012).

Bejarano-Guzmán, Ramiro, Procesos declarativos, ejecutivos y arbitrales ( $5^{\mathrm{a}}$ ed., Editorial Temis, Bogotá, 2011).

CAlamandrei, Piero, Introducción al estudio sistemático de las providencias cautelares (Editorial Bibliográfica Argentina, Buenos Aires, 1984).

Couture, Eduardo J., Fundamentos del derecho procesal civil (Editorial B de F Ltda., Buenos Aires, 2010).

Etcheverry, Raúl, Derecho comercial y económico (Editorial Astrea, Buenos Aires, 2005).

Fraga-Pittaluga, Luis, El arbitraje en el derecho administrativo (Los Talleres Gráficos de Editorial Torino, Caracas, 2000).

González-López, Édgar \& Herrera-Zapata, Luz Mónica, Análisis de laudos arbitrales en derecho de las telecomunicaciones (Editorial Externado de Colombia, Bogotá, 2012).

Hart, Her bert, El concepto del derecho (Editorial Abeledo-Perrot, Buenos Aires, 2009).

López-Blanco, Hernán Fabio, La jurisprudencia arbitral en Colombia, Tomo IV (Editorial Universidad Externado de Colombia, Bogotá, 2007).

Marinoni, Luiz Guilherme; Pérez-Ragone, Álvaro \& Núñez-Ojeda, Raúl, Fundamentos del Proceso Civil. Hacia una teoría de la adjudicación (Editorial Abeledo Perrot, Santiago de Chile, 2010).

Quintero, Beatriz \& Prieto, Eugenio, Teoría general del derecho procesal (4 ${ }^{\mathrm{a}}$ ed., Editorial Temis, Bogotá, 2008).

Reglero-Campos, Fernando, El arbitraje: el convenio arbitral y las causas de nulidad del laudo en la ley de 5 de diciembre de 1988 (Montecorvo, Madrid, 1991).

Rodríguez-Mejía, Marcela, Medidas cautelares en el proceso arbitral (Editorial Externado de Colombia, Bogotá, 2013).

Zapata de Arbeláez, Adriana; Barona-Vilar, Silvia \& Esplugues-Mota, Carlos, El arbitraje interno e internacional en Latinoamérica. Regulación presente y tendencias de futuro (Editorial Externado de Colombia, Bogotá, 2010). 


\section{Contribuciones en obras colectivas}

Alvarado-Velloso, Adolfo, El procedimiento cautelar y la solución urgente de una pretensión, en Memorias del XXXIV Congreso Colombiano de Derecho Procesal, 834-888 (Instituto Colombiano de Derecho Procesal, ICDP, Medellín, 2013). Disponible en: https://letrujil.files.wordpress.com/2013/09/35adolfo-alvaradovelloso.pdf

Klett-Fernández, Selva Anabella, Medidas cautelares innominadas en el Código General del Proceso en la República de Uruguay, en Memorias del XXXIV Congreso Colombiano de Derecho Procesal, 996-1039 (Instituto Colombiano de Derecho Procesal, Medellín, 2013). Disponible en: https://letrujil.files.wordpress. com/2013/09/40selva-anabella-klett-f.pdf

Marinoni, Luiz Guilherme, La evolución de la técnica anticipatoria y de la tutela preventiva en Brasil, en Memorias del XXXIV Congreso Colombiano de Derecho Procesal, Medellín, 11, 12 y 13 de septiembre de 2013, 975-993 (Universidad Pontificia Bolivariana, Medellín, 2013). Disponible en: https://docs. lejb25ncmVzb2RjaG9wcm9jZXNhbHxneDo2NmNkM2Q1MGUwMWZhODAy

Morello, Augusto Mario \& Véscovi, Enrique, Medidas cautelares y medidas provisionales, en Medidas provisionales y medidas cautelares en el Sistema Interamericano de Derechos Humanos, 147-203 (ERnESTo ReY-CANTOR \& Ángela Rey-AnAyA, Instituto Interamericano de los Derechos Humanos. México, 1996). Disponible en: http://biblio.juridicas.unam.mx/libros/libro.htm?l=2047, http:// biblio.juridicas.unam.mx/libros/5/2047/12.pdf

Nieva-Fenoll, Jordi, El elemento psicológico en la adopción de las medidas cautelares, en Memorias del IX Congreso Internacional de Derecho Procesal, San José de Cúcuta, 2 y 3 de agosto de 2013, 107-128 (Universidad Libre Seccional Cúcuta, Cúcuta, 2013). Disponible en: http://portales.te.gob.mx/seminario/sites/portales. te.gob.mx.seminario/files/materiales/Jordi\%20Nieva.pdf

Parra-Quijano, JaIro, Medidas cautelares innominadas, en Memorias del XXXIV Congreso Colombiano de Derecho Procesal, 300-318 (Instituto Colombiano de Derecho Procesal, ICDP, Medellín, 2013). Disponible en: https://letrujil.files. wordpress.com/2013/09/12jairo-parra-quijano.pdf

\section{Revistas}

Cremades, Bernardo M. \& Madalena, Ignacio, Procedimientos paralelos en el arbitraje internacional, 6 Revista Peruana de Arbitraje, 3-88 (2008). Disponible en: http:// www.peruarbitraje.org/pdf/revista/REVISTA_PERUANA_DE_ARBITRAJE_ RPA_6_2008.pdf

Horning, Richard Allan, Interim Measures of Protection; Security for Claims and Costs, and Commentary on the WIPO Emergency Relief Rules, 9 American Review of International Arbitration, 1-4 (1998).

Perera, Pedro \& Angrisano, Humberto, Las medidas cautelares en el arbitraje, Revista Comité de Arbitraje (2013). Disponible en: http://www.venamcham.org/index. php?option=com_content $\&$ id $=1296 \% 3$ Acomite-de-arbitraje-las-medidas- 
cautelares-en-el-arbitraje-febrero-2013\&Itemid=19

Rangel-Romberg, Aristides, Medidas cautelares innominadas, 8 Revista del Instituto Colombiano de Derecho Procesal, ICDP, 8, 85-111 (1989). Disponible en: http:// publicacionesicdp.com/index.php/Revistas-icdp/article/view/293/pdf

\section{Artículos web}

Díaz-Vallejos, José W., Apuntes sobre la medida cautelar. Disponible en: http:// asociacionjusticiayderechouigv.blogspot.com.co/2009/12/algunos-apuntessobre-la-medida.html, http://asociacionjusticiayderechouigv.blogspot. com/2009/12/algunos-apuntes-sobre-la-medid

Fraga-Lo Curto, Luis Ángel, Las medidas cautelares anticipadas en el arbitraje venezolano, Centro Empresarial de Conciliación y Arbitraje, CEDCA. Disponible en: http://www.cedca.org.ve/documentos/Luis-Fraga.pdf

Sartori, Giovanni, El debido concepto de lo cautelar. Disponible en: http://www. academiadederecho.org/upload/biblio/contenidos/SARTORI.pdf, http://www. academiadederecho.org/upload/biblio/contenidos/SARTORI

Subirats-Aleixandri, María Cinta, Las medidas cautelares: su instrumentalidad. Disponible en: http://www.derecho.com/articulos/2001/07/15/las-medidascautelares-su-instrumentalidad/

\section{Tratados internacionales y legislación sobre arbitraje}

Cámara de Comercio Internacional, CCI, Reglamento de Arbitraje de la Cámara de Comercio Internacional, CCI (2012). Disponible en: http://www.sice.oas.org/ dispute/comarb/icc/arbruls.asp, http://www.iccwbo.org/Data/Documents/ Buisness-Services/Dispute-Resolution-Services/Mediation/Rules/2012Arbitration-Rules-and-2014-Mediation-Rules-SPANISH-version/

Estados Unidos, Asociación Americana de Arbitraje, AAA, Reglamento de arbitraje internacional de la Asociación Americana de Arbitraje, AAA (1997). Texto disponible en español e inglés: http://www.intracen.org/Reglamento-deArbitraje-Internacional-de-la-Asociacion-Americana-de-Arbitraje-1997/, http://www.intracen.org/International-Arbitration-Rules-of-the-AmericanArbitration-Association-2001/

Inglaterra, Corte de Arbitraje Internacional de Londres, LCIA, Reglamento de la Corte (1998). Disponible en: http://www.lcia.org/Dispute_Resolution_Services/ LCIA_Arbitration_Rules.aspx

Instituto Internacional para la Unificación del Derecho Privado, UNCITRAL, Principios Unidroit sobre los Contratos Comerciales Internacionales, Roma, 2004. Disponible en: http://www.unidroit.org/spanish/principles/contracts/ principles2004/integralversionprinciples2004-s.pdf

Italia, Associazione Italiana per l'Arbitrato, AIA, Asociación Italiana para Arbitraje, Reglamento de Asociación Italiana para Arbitraje. Disponible en: http:// arbitratoaia.com/images/immagini/Regolamento_Arbitrato_AIA_2016.pdf 
México, Centro de Arbitraje de México, CAM, Reglamento del Centro de Arbitraje de México, CAM (2009). Disponible en: http://www.camex.com.mx/index.php/ arbitraje-cam/reglas-de-arbitraje-del-cam

Organización Mundial de la Propiedad Intelectual, OMPI, Reglamento de Arbitraje del Tribunal de la Organización Mundial de la Propiedad Intelectual, OMPI. Disponible en: http://www.wipo.int/amc/es/arbitration/rules/, http://www.wipo. int/amc/en/arbitration/rules/

Venezuela, Centro Empresarial de Conciliación y Arbitraje, CEDCA, Reglamento de Conciliación y Arbitraje. Disponible en: http://www.cedca.org.ve/documentos/ normativa_arbitraje/iii.\%20Reglamento\%20CEDCA.pdf

\section{Normatividad internacional}

España, Ley 1/2000, 7 de enero, Ley de Enjuiciamiento Civil. Disponible en: https:// boe.es/buscar/act.php?id=BOE-A-2000-323

Francia, Loi 2007-1787, Nouveau Code de Procédure civile, 20 décembre 2007. Disponible en: https://www.legifrance.gouv.fr/affichCode.do?cidTexte=LEGI TEXT000006070716

Inglaterra, Ley de Arbitraje Inglesa de 1996. Disponible en: http://www.legislation.gov. uk/ukpga/1996/23/contents

Suecia, Ley de Arbitraje, 4 de marzo de 1999. Disponible en: http://www.sc.ehu.es/ dpwlonaa/legislacion/ARBITRAJE\%20INTERNACIONAL/Ley\%20Sueca/ Ley\%20sueca.htm, http://www.uni-kiel.de/leobalt/Datenbank/Schweden/ The $\% 20$ Swedish $\% 20$ Arbitration $\% 20$ Act $\% 20$ of $\% 201999$.htm

\section{Normatividad colombiana}

Colombia, Constitución Política, 7 de julio de 1991. Versión corregida, 116 Gaceta Constitucional, 20 de julio de 1991, disponible en: http://www.secretariasenado. gov.co/senado/basedoc/constitucion_politica_1991.html

Colombia, Decreto 2651 de 1991, por el cual se expiden normas transitorias para descongestionar los despachos judiciales, 40.177 Diario Oficial, 25 de noviembre de 1991. Disponible en: http://www.alcaldiabogota.gov.co/sisjur/normas/Norma1. jsp?i=14319

Colombia, Decreto 1818 de 1998, por medio del cual se expide el Estatuto de los mecanismos alternativos de solución de conflictos, 43.380 Diario Oficial, 7 de septiembre de 1998. Disponible en: http://www.alcaldiabogota.gov.co/sisjur/ normas/Norma1.jsp?i=6668

Colombia, Ley 57 de 1887, Código Civil, 26 de mayo de 1873. Disponible en: http://www. secretariasenado.gov.co/senado/basedoc/codigo_civil.html

Colombia, Ley 105 de 1890, sobre reformas a los procedimientos judiciales, 8.296 Diario Oficial, 7 de enero de 1891. Disponible en: http://www.icbf.gov.co/cargues/avance/ docs/ley_0105_1890.htm 
Colombia, Ley 103 de 1923, sobre organización judicial y procedimiento civil, 19.498 a 19.509 Diario Oficial, 18 de febrero de 1924. Disponible en: http://www.cancilleria. gov.co/sites/default/files/Normograma/docs/ley_0103_1923.htm

Colombia, Ley 28 de 1931, sobre cámaras de comercio, 21.624 Diario Oficial, 23 de febrero de 1931. Disponible en: http://bibliotecadigital.ccb.org.co/bitstream/ handle/11520/14013/Ley\%2028\%20de \%201931.pdf;sequence=1

Colombia, Ley 105 de 1931, sobre organización judicial y procedimiento civil, 21.823 Diario Oficial, 24 de octubre de 1931. Disponible en: http://icbf.gov.co/cargues/ avance/docs/ley_0105_1931.htm

Colombia, Ley 23 de 1991, por medio de la cual se crean mecanismos para descongestionar los despachos judiciales, y se dictan otras disposiciones, 39.752 Diario Oficial, 21 de marzo de 1991. Disponible en: http://www.alcaldiabogota.gov.co/sisjur/ normas/Norma1.jsp?i=6546\#1

Colombia, Ley 256 de 1996, por medio de la cual se dictan normas sobre competencia desleal, 42.692 Diario Oficial, 18 de enero de 1996. Disponible en: http://www. secretariasenado.gov.co/senado/basedoc/ley_0256_1996.html

Colombia, Ley 446 de 1998, por la cual se adoptan como legislación permanente algunas normas del Decreto 2651 de 1991, se modifican algunas del Código de Procedimiento Civil, se derogan otras de la Ley 23 de 1991 y del Decreto 2279 de 1989, se modifican y expiden normas del Código Contencioso Administrativo y se dictan otras disposiciones sobre descongestión, eficiencia y acceso a la justicia, 43.335 Diario Oficial, 8 de julio de 1998. Disponible en: http://www. secretariasenado.gov.co/senado/basedoc/ley_0446_1998.html

Colombia, Ley 1285 de 2009, por medio de la cual se reforma la Ley 270 de 1996 Estatutaria de la Administración de Justicia, 47.240 Diario Oficial, 22 de enero de 2009. Disponible en: http://www.secretariasenado.gov.co/senado/basedoc/ ley_0446_1998.html

Colombia, Ley 1563 de 2012, por medio de la cual se expide el Estatuto de Arbitraje Nacional e Internacional y se dictan otras disposiciones, 48.489 Diario Oficial, 12 de julio de 2012. Disponible en: http://www.secretariasenado.gov.co/senado/ basedoc/ley_1563_2012.html

Colombia, Ley 1564 de 2012, por medio de la cual se expide el Código General del Proceso y se dictan otras disposiciones, 48.489 Diario Oficial, 12 de julio de 2012. Disponible en: http://www.secretariasenado.gov.co/senado/basedoc/ ley_1564_2012.html

\section{Casos internacionales}

Francia, Cour de cassation, asunto Société d'exploitation du Cinema Rex v. Société Rex, 7 de junio de 1979. 


\section{Jurisprudencia colombiana}

Corte Constitucional, Expediente D-4974, Sentencia C-379-04, 27 de abril de 2004, magistrado ponente Alfredo Beltrán-Sierra. Disponible en: http://www. corteconstitucional.gov.co/relatoria/2004/C-379-04.htm

Corte Constitucional, expediente D-9626, Sentencia C-835-13, 20 de noviembre de 2013, magistrado ponente Nilson Pinilla-Pinilla. Disponible en: http://www. corteconstitucional.gov.co/relatoria/2013/c-835-13.htm 
